\title{
Estratégias autoprejudiciais em matemática: uma revisão sistemática da literatura
}

\author{
Self-handicapping strategies in mathematics: a
}

systematic review of literature

Estrategias auto-perjudiciales en matemáticas: una

revisión sistemática de la literatura

EDMILSON MINORU TORISU

EVELY BORUCHOVITCH (iD)

\section{Resumo}

A adoção de estratégias autoprejudiciais de aprendizagem envolve a criação ou declaração de obstáculos, reais ou fictícios, antes da realização de uma tarefa para justificar, antecipadamente, um possível fracasso ou para tornar um sucesso mais recompensador para a autoimagem. Esta prática pode afetar o desempenho e ter consequências desastrosas para o estudante. No caso da Matemática, disciplina temida por muitos, a importância de se pesquisar a influência destas estratégias na vida dos estudantes parece ser ainda maior. Assim, o presente artigo tem como objetivo apresentar uma revisão sistemática da literatura que investiga a adoção de estratégias autoprejudiciais no âmbito da Matemática. A busca foi realizada nas bases de dados SciELO, PsycINFO e ERIC. A amostra final incluiu 10 textos, todos internacionais, sendo 3 trabalhos em eventos e 7 artigos em periódicos. Os resultados revelaram que vários fatores contribuem para a adoção, ou não, de estratégias autoprejudiciais em Matemática. $\mathrm{O}$ ambiente de sala de aula e o apoio que o professor oferece a seus alunos, bem como a importância de se considerar outros construtos, como a autoestima e autoeficácia, como algo que afeta a escolha pelo uso de estratégias autoprejudiciais foram variáveis-chave para maior compreensão da adoção destas estratégias. 0 pequeno número de pesquisas encontrado no âmbito da Matemática sugere que este é ainda um campo profícuo para novas pesquisas, tanto brasileiras quanto internacionais.

\footnotetext{
a Universidade Federal de Ouro Preto (UFOP), Ouro Preto, MG, Brasil. Doutor em Educação, e-mail: etorisu@gmail.com

b Universidade Estadual de Campinas (UNICAMP), Campinas, SP, Brasil. Doutora em Educação, e-mail: evely@unicamp.br
} 
Palavras-chave: Estratégias autoprejudiciais. Autorregulação. Matemática.

\begin{abstract}
The adoption of self-handicapping learning strategies involves creating or declaring obstacles, real or fictitious, before carrying out a task to justify, in advance, a possible failure or to make a success more rewarding for self-image. This practice can affect performance and have disastrous consequences for the student. In the case of Mathematics, a subject feared by many, the importance of researching the influence of these strategies on the students' lives seems to be even greater. Thus, this article aims to present a systematic review of the literature that investigates the adoption of selfhandicapping strategies in the field of Mathematics. The search was carried out in the SCIELO, PSYCINFO and ERIC databases. The final sample included 10 texts, all international, with 3 papers in events and 7 articles in journals. The results revealed that several factors contribute to the adoption, or not, of self-handicapping strategies in Mathematics. The classroom environment and the support that the teacher offers to his students, as well as the importance of considering other constructs, such as self-esteem and self-efficacy as something that affects the choice to use self-handicapping strategies were key variables for greater understanding. adoption of these strategies. The small number of researches found in the field of Mathematics suggests that this is still a fruitful field for new research, both Brazilian and international.
\end{abstract}

Keywords: Self-handicapping strategies. Self-regulation. Math.

\title{
Resumen
}

La adopción de estrategias de aprendizaje auto-perjudiciales implica crear o declarar obstáculos, reales o ficticios, antes de realizar una tarea para justificar, de antemano, un posible fracaso o hacer más gratificante un éxito para la autoimagen. Esta práctica puede afectar el desempeño y tener consecuencias desastrosas para el estudiante. En el caso de las Matemáticas, disciplina temida por muchos, la importancia de investigar la influencia de estas estrategias en la vida de los estudiantes parece ser aún mayor. Así, este artículo tiene como objetivo presentar una revisión sistemática de la literatura que investiga la adopción de estrategias auto-perjudiciales en el campo de las Matemáticas. La búsqueda se realizó en las bases de datos SCIELO, PSYCINFO y ERIC. La muestra final incluyó 10 textos, todos internacionales, con 3 artículos en eventos y 7 artículos en revistas. Los resultados revelaron que varios factores contribuyen a la adopción, o no, de estrategias auto-perjudiciales en Matemáticas. El ambiente del aula y el apoyo que el docente brinda a sus alumnos, así como la importancia de considerar otros constructos, como la autoestima y la autoeficacia, como algo que incide en la elección de utilizar estrategias auto-perjudiciales, fueron variables clave para Mayor comprensión Adopción de estas estrategias. El pequeño número de investigaciones encontradas en el campo de las Matemáticas sugiere que este sigue siendo un campo fructífero para nuevas investigaciones, tanto brasileñas como internacionales.

Palabras clave: Estrategias auto-perjudiciales. Autorregulación. Matemáticas. 


\section{Introdução}

Um dos papéis da escola, em uma sociedade multicultural, é desenvolver nos estudantes várias competências que permitam a eles gerir seus processos de aprendizagem e trilhar, com autonomia, sua vida acadêmica, fortalecendo suas capacidades individuais e transformando-as em habilidades necessárias ao seu projeto de vida (ZIMMERMAN; SCHUNK, 2011; PRANKE; FRISON, 2015; GOMES; BORUCHOVITCH, 2020). Entretanto, essa não é uma tarefa simples. Há muitos aspectos envolvidos e imbricados no processo educativo que podem influenciar a maneira como os estudantes aprendem. Dentre eles, está a capacidade desses atores de serem autorreguladores de suas aprendizagens.

A autorregulação da aprendizagem pode ser definida como o processo de monitorar, controlar e refletir sobre o próprio aprendizado (ZIMMERMAN; SCHUNK, 2011). No contexto educacional, autorregular a aprendizagem contribui para que o estudante assuma, em boa medida, o controle do seu processo de aprendizagem. Além de melhorar o desempenho acadêmico dos estudantes, a autorregulação da aprendizagem tem reflexos positivos na maneira como eles, ao longo de suas vidas, administrarão as demandas de aprendizagens. Todavia, a autorregulação da aprendizagem precisa ser aprendida na escola e desenvolvida ao longo da vida. Sendo assim, a escola e, em particular, os professores assumem papel fundamental para que os estudantes desenvolvam estratégias autorregulatórias que promovem a aquisição do seu conhecimento por meio de estratégias de aprendizagem e o fomento de crenças motivacionais e estados emocionais adequados à aprendizagem (GANDA; BORUCHOVITCH, 2019; SCHUNK; GREENE, 2018; ZIMMERMAN; SCHUNK, 2011).

No início, de acordo com Boruchovitch (2014), baseada em Zimmerman e Schunk (2011), as pesquisas exploravam, basicamente, as estratégias de aprendizagem. Com o passar do tempo, os pesquisadores perceberam que a utilização destas estratégias era afetada por fatores motivacionais, de metas, de atribuições de causalidade, autoeficácia, emoções, dentre outros. Com o passar do tempo, vários autores (SCHUNK; ZIMMERMAN, 2008; SCHUNK; GREENE, 2018; ZIMMERMAN; SCHUNK, 2011) passam a compreender a autorregulação como um 
constrtuto que incorpora a relação entre quatro dimensões: cognitiva/metacognitiva, motivacional, emocional/afetiva e social.

A dimensão cognitiva/metacognitiva envolve as estratégias de aprendizagem dos alunos e sua posterior avaliação, por meio da reflexão. A dimensão motivacional envolve o estudo, por exemplo, das crenças do estudante sobre suas capacidades, como as crenças de autoeficácia, entre outras. Os estados emocionais podem afetar positiva ou negativamente o desempenho de estudantes antes, durante e depois de uma atividade (PEKRUN, 2006; 2018). A dimensão social é defendida por muitos estudiosos da autorregulação por entenderem que, se o indivíduo está inserido em um meio, este terá influência sobre suas ações (HAWDIN; JÄRVELÄ; MILLER, 2018).

Vários são os estudiosos que têm se dedicado a pesquisar a autorregulação da aprendizagem (MCCASLIN; GOOD, 1996; PINTRICH 2000, 2004; ZIMMERMAN, 2000; ZIMMERMAN; SCHUNK, 2011; BORUCHOVITCH, 2014) e seu potencial para contribuir para o sucesso acadêmico dos estudantes. Como consequência, a literatura apresenta vários modelos teóricos de autorregulação, como o de Bandura (1978), Zimmerman (2000), Schunk (2001), Pintrich (2000, 2004), McCaslin; Good (1996), Winne; Hadwin (1998); Boekaerts (1991, 1992), Efklides (2011), dentre outros.

Um dos mais importantes e conhecidos desses modelos é o de Zimmerman (2000), baseado na TSC (BANDURA 1978; 1986) e no qual concebe a autorregulação como tendo três fases cíclicas. A fase de antecipação ou previsão é aquela na qual o estudante analisa e avalia a tarefa. A fase de controle de desempenho ou de volição é aquela na qual o estudante põe em prática ações e comportamentos para resolver a tarefa. Nesta fase emergem várias estratégias de aprendizagem. $\mathrm{Na}$ terceira fase, denominada de autorreflexão, o estudante avalia criticamente as estratégias de aprendizagem empregadas na fase anterior, bem como os resultados obtidos referentes à realização da tarefa. Nesta última fase, caso o estudante tenha adotado estratégias de aprendizagem que contribuíram para o seu sucesso, ele as avaliará como positivas e isso pode contribuir para o surgimento de sentimentos de satisfação. Caso as estratégias tenham levado o estudante ao fracasso, elas serão avaliadas negativamente e sentimentos de incapacidade poderão surgir (GANDA; BORUCHOVITVH, 2016; ZIMMERMAN, 2000). O sentimento de fracasso poderá 
suscitar a adoção, pelo estudante, de comportamentos que prejudicam sua aprendizagem como, por exemplo, o uso de estratégias autoprejudiciais, foco principal do presente artigo.

A expressão "estratégias autoprejudiciais", empregada neste texto, é uma tradução para o português da expressão, em inglês, self-handcapping strategies (BERGLASS; JONES, 1978; MIDGLEY; ARUNKUMAR; URDAN, 1996), academic self-handcapping (LEONDARI; GONIDA, 2007), claimed selfhandcapping strategies (COUDEVYLLE et al., 2020) ou avoidance strategies (TURNER et al., 2002).

Berglas e Jones (1978, p. 406, tradução nossa) definem estratégias autoprejudiciais como "qualquer ação ou escolha de configuração de desempenho que aumenta a oportunidade de externalizar (ou desculpar) o fracasso e de internalizar (aceitar o crédito pelo) sucesso”. Estratégias autoprejudiciais declaradas (claimed selfhandcapping strategies) "envolvem declarar um ou vários impedimentos reais ou fictícios antes de uma apresentação, para justificar antecipadamente um possível fracasso ou para tornar um sucesso mais recompensador para a autoimagem" (COUDEVYLLE et al., 2020, p. 1, tradução nossa).

Embora haja várias maneiras de se compreender as estratégias autoprejudiciais, Sameer Babu e Selvamari (2018) consideram que a maioria dos autores concorda que elas envolvem a criação de obstáculos para o desempenho bem-sucedido em tarefas que o indivíduo considera importantes. Como o obstáculo pode interferir no desempenho, ele passa a ser a razão (assume a culpa) para um mau desempenho, eliminando a responsabilidade do indivíduo pelo fracasso. Esta ideia está implícita em parte do título do artigo intitulado If I don't do well tomorrow, there's a reason: predictors of adolescents' use of academic self-handcapping strategies (MIDGLEY; ARUNKUMAR; URDAN, 1996). A parte negritada traduz muito bem o pensamento de quem costuma criar obstáculos para justificar maus desempenhos. Os autores do artigo afirmam que, no âmbito escolar, alguns alunos procrastinam, brincam, diminuem o esforço e usam outras estratégias autoprejudiciais para que, caso o desempenho subsequente seja baixo, estas circunstâncias sejam tidas como causa.

Garcia et al. (1985, apud MIDGLEY; ARUNKUMAR; URDAN, 1996) acreditam que estudantes precisam ter atingido uma idade em que tenham capacidade 
cognitiva para compreender a diferença entre inteligência, capacidade e esforço para que possam utilizar estratégias autoprejudiciais intencionalmente. Por conseguinte, este uso é mais comum entre estudantes a partir do ensino fundamental e pode, também, ter relação com o gênero. Yu e McLellan (2019) realizaram uma pesquisa com 536 estudantes na Inglaterra, com idades entre 13 e 14 anos, com o objetivo de identificar diferenças na motivação acadêmica e social de adolescentes de ambos os sexos, bem como examinar o papel desses construtos na explicação das diferenças de gênero no que se refere ao uso de estratégias autoprejudiciais. Os resultados evidenciaram que os meninos adotam com maior frequência estas estratégias porque maniestam desejo mais forte de preservar sua imagem nos domínios acadêmico e social, quando comparados ao sexo feminino.

Há tipos distintos de estratégias autoprejudiciais. Elas podem ser comportamentais ou verbais. Arkin e Baumgardner (1985, apud LEONDARI; GONIDA, 2007), utilizaram as expressões impedimentos adquiridos (acquired impediments), que podem diminuir as chances de sucesso do indivíduo, e impedimentos declarados (claimed impediments), que ocorrem quando o indivíduo declara uma condição autoprejudicial para nomear as estratégias autoprejudicias adquiridas e verbais, respectivamente. Leary e Shepperd (1986, apud LEONDARI; GONIDA, 2007), adotaram, para as mesmas expressões, os termos comportamento autoprejudicial (behavioural self-handcapping) e autodeclaração da estratégia autoprejudicial (self-reported handcapping). Como exemplos de estratégias autoprejudicias relacionadas ao comportamento, pode-se citar as que se seguem: falta de sono, consumo de drogas e álcool, escolha de circunstâncias debilitantes de desempenho, redução estratégica do esforço, pouco ou nenhum esforço antes ou durante as tarefas. Para as estratégias autoprejudiciais declaradas, tem-se como exemplos: ansiedade social, ansiedade antes de testes, sintomas físicos e psicológicos, incluindo alegação de problemas de saúde (LEONDARI; GONIDA, 2007).

Estes tipos de estratégias autoprejudiciais diferem uns dos outros em termos de possíveis prejuízos para o indivíduo. As estratégias autoprejudiciais declaradas são menos prejudiciais do que as referentes ao comportamento, pois não reduzem necessariamente a probabilidade de um desempenho bem-sucedido. Um exemplo está relacionado ao consumo de álcool. Se o consumo ocorrer antes de uma situação 
importante, servirá como justifictiva para um mau desempenho, mas também diminuirá as chances de um desempenho bem-sucedido. Caso o indivíduo simplesmente relate estar ansioso, isto servirá de justificativa para um desempenho ruim, mas sem diminuir as chances de sucesso (HIRT; DEPPE; GORDON, 1991).

A adoção de estratégias autoprejudiciais por um indivíduo revela que ele se preocupa com os julgamentos dos outros frente ao seu desempenho (GANDA; BORUCHOVITCH, 2016; URDAN; MIDGLEY, 2001), compreendidos como uma imagem de si mesmo (SHEPPARD; ARKIN, 1989). Elas servem, portanto, como um mecanismo de autoproteção porque nossa sociedade valoriza muito os indivíduos em função de sua capacidade de realização (COVINGTON, 2000; ŞAHIN; ÇOBAN, 2020, COUDEVYLLE et al., 2020).

De forma semelhante à autoproteção como mecanismo de defesa dos julgamentos alheios, a autovalorização (self-worth) também pode desencadear a adoção de estratégias autoprejudiciais (COVINGTON, 2000). O autor teoriza que a autovalorização baseia-se na crença de que o comportamento dos estudantes nas escolas pode também ser compreendido em termos de suas tentativas para manter uma autoimagem positiva, evitando parecer incompetente. A procrastinação é um dos caminhos adotados para isso.

Os procrastinadores se colocam em uma posição defensiva em que sempre estão a salvo dos maus julgamentos. Se vão mal, podem dizer que é porque adiaram os estudos até o último momento. Se eles se saírem bem, apesar de procrastinar, os outros os perceberão como particularmente capazes. Covington (2000) também descreve os alunos que se envolvem em muitas atividades para que possam utilizar isso como uma razão para o mau desempenho, caso ocorra.

Pesquisas evidenciam que pessoas com baixa autoestima fazem mais uso de estratégias autoprejudiciais (ZIMMERMAN, 2000; PRAPAVESSIS; GROVE, 1998; CHEN; SUNG; WANG, 2017; YAVUZER, 2015). Por outro lado, o uso de estratégias autoprejudiciais, embora possa camuflar as razões para um mau desempenho, afeta as pessoas de muitas formas. De acordo com Leondari e Gonida (2007), a adoção destas estratégias pode levar o estudante a um baixo rendimento acadêmico, a evitar comportamental e mentalmente os trabalhos escolares, a uma percepção pessimista do desempenho acadêmico e, às vezes, à diminuição da 
autoestima. Parece, então, que este é um sistema que se retroalimenta, gerando um perigoso ciclo vicioso. A baixa autoestima leva ao uso das estratégias autoprejudiciais e estas, por sua, vez, trazem como consequência diminuição da autoestima, bem como redução na aprendizagem (ZUCKERMAN; KIEFFER; KNEE, 1998; MIRANDA; BORUCHOVITCH; GANDA， 2017; ZIMMERMAN， 2000; SCHUNK; ZIMMERMAN, 2008).

Outro aspecto importante a ser ressaltado acerca das estratégias autoprejudiciais é que elas estão associadas às características particulares dos alunos, como suas motivações gerais e atitudes em relação à aprendizagem. O ambiente também é outro aspecto a ser considerado. De acordo com Sameer Babu e Selvamari (2018), as escolas e salas de aula se configuram como excelentes contextos para examinar estas estratégias, já que nelas os estudantes são confrontados com tarefas e situações em que as informações sobre sua capacidade e inteligência podem ser discutidas e visualizadas por todos, particularmente aquelas que envolvem algum tipo de diagnóstico de habilidades.

Estudantes com histórico de baixo rendimento terão, gradativamente, expectativa de desempenho bem-sucedido menores, especialmente se acreditarem que a falha é resultado de causas específicas estáveis e incontroláveis, como falta de inteligência ou capacidade. A partir do momento em que os indivíduos desenvolvem a crença de que podem falhar em uma tarefa futura, eles se tornam mais propensos à utilização de estratégias autoprejudiciais, que podem comprometer os seus planos acadêmicos. Este ciclo de fracasso-estratégias-fracasso pode levar o estudante a desisitir de empreender esforços no contexto educativo (URDAN; MIDGLEY, 2003; 2001). Esse ciclo vicioso é muito semelhante ao mencionado anteriormente, em relação à autoestima.

Como a adoção de estratégias autoprejudiciais traz consequências não desejáveis ao estudante nos aspectos cognitivos, motivacionais, afetivos e sociais, e a literatura nacional sobre esta importante variável ainda é incipiente, o objetivo do presente artigo é apresentar uma revisão sistemâtica da literatura acerca de estudos que tenham investigado o uso estratégias autoprejudiciais em salas de aula de Matemática. Esta escolha está relacionada ao fato de as pesquisas mostrarem que a Matemática, enquanto disciplina escolar, é algo temido e que "ocupa um espaço no 
imaginário coletivo que, muitas vezes, predispõe as pessoas a esperar pelo fracasso. As crenças dominantes destacam esta disciplina como 'muito importante', 'essencial para a vida', mas 'muito difícil', 'destinada apenas para poucos"' (TORISU; FERREIRA, 2009, p. 169). Esta forma de perceber a Matemática como algo ameaçador e de difícil compreensão, para muitos estudantes ao redor do mundo, pode ser a causa da adoção de estratégias autoprejudiciais.

\section{Método}

Foi realizada uma revisão sistemática da literatura sobre uso de estratégias autoprejudiciais relacionadas à Matemática. As buscas foram realizadas no mês de fevereiro de 2021, utilizando as palavras-chave "estratégias autoprejudiciais" e "Matemática"; "self-handcapping" e "mathematics"; "avoidance strategies" e "mathematics" em três bases de dados: SciELO, PsycINFO e ERIC. Há muitas pesquisas cujo foco está nas estratégias autoprejudicias ou nas quais este construto é relevante. Contudo, nossa hipótese era de que trabalhos que abordassem estas estratégias em Matemática seriam poucos. A partir disso, imaginaram-se duas possibilidades de movimentos de busca: 1 - caso fossem encontradas muitas pesquisas com foco nas estratégias autoprejudiciais em Matemática, faríamos um recorte temporal dos últimos cinco anos (2016 - 2020); 2 - caso o conjunto de pesquisas fosse pequeno, todas elas seriam consideradas para esta revisão. Após realizarmos as buscas e constatarmos um pequeno grupo de pesquisas, optamos por revisitar todas. Foram considerados somente artigos completos em periódicos ou trabalhos completos publicados em anais de aventos.

$\mathrm{Na}$ busca realizada na base de dados SciELO, não foram encontradas pesquisas utilizando as palavras-chave citadas anteriormente. Na plataforma da PsycINFO, o total de trabalhos encontrados foi o seguinte: com as palavras-chave: "estratégias autoprejudiciais" e "matemática" = 0 ; "self-handcapping" e "mathematics" = 0; "avoidance strategies" e "mathematics" $=2$. Em um desses dois trabalhos, as palavras-chave apareceram no título e, no outro, no resumo, o que nos levou a considerá-los, também. Na base de dados da plataforma ERIC, foram encontrados 12 trabalhos. Desses 12, um já havia sido encontrado na busca realizada 
na plataforma PsycINFO, isto é, um título estava repetido. Outro, embora com título diferente, apresentava os resultados da mesma pesquisa. Um terceiro foi descartado por apresentar as estratégias prejudiciais em uma perspectiva diferente daquela que nos interessa, qual seja, aquela relacionada à Matemática. Sendo assim, a plataforma ERIC nos forneceu nove trabalhos no total. Desses nove, em três, as palavras-chave "self-handicapping" e "mathematics" ou "math", aparecem explicitamente no título; em dois, a palavra-chave "self-handicapping" aparece no título, mas "mathematics" aparece somente no resumo; em três, acontece o contrário: a palavra-chave mathematics está presente no título e "self-handicapping" somente no resumo; em um trabalho, aparecem no título as palavras-chave "avoidance strategies" e "mathematics". Apenas em um artigo as palavras-chave aparecem somente no resumo. Assim, no total, foram analisadas 10 pesquisas, 1 da plataforma PsycINFO e 09 da plataforma ERIC.

\section{Resultados e discussão}

Os trabalhos selecionados serão primeiramente apresentados em um quadro resumo (quadro 1), com informações gerais. Em seguida, informações mais detalhadas acerca de cada um serão dadas e analisadas. 
Quadro 1 - informações gerais sobre as pesquisas selecionadas

\begin{tabular}{|c|c|c|c|c|}
\hline Ano & $\begin{array}{l}\text { Periódico } \\
\text { (P) ou } \\
\text { Evento } \\
\text { (E) }\end{array}$ & $\begin{array}{l}\text { País do } \\
\text { periódico } \\
\text { ou do } \\
\text { evento }\end{array}$ & $\begin{array}{l}\text { Nome do periódico ou } \\
\text { do evento }\end{array}$ & Título do trabalho \\
\hline 1993 & $E$ & $\begin{array}{l}\text { Estados } \\
\text { Unidos }\end{array}$ & $\begin{array}{l}\text { Annual Meeting of the } \\
\text { American Educational } \\
\text { Research Association }\end{array}$ & $\begin{array}{l}\text { An Assessment of the Self- } \\
\text { Protective Function of Self- } \\
\text { Handicapping }\end{array}$ \\
\hline 2000 & $E$ & $\begin{array}{l}\text { Estados } \\
\text { Unidos }\end{array}$ & $\begin{array}{l}\text { Annual Meeting of the } \\
\text { Southeastern } \\
\text { Psychological Association }\end{array}$ & $\begin{array}{c}\text { Science Anxiety as a Function } \\
\text { of Personality, Gender Roles, } \\
\text { Experience with Science }\end{array}$ \\
\hline 2001 & $E$ & $\begin{array}{l}\text { Estados } \\
\text { Unidos }\end{array}$ & $\begin{array}{l}\text { Annual Meeting of the } \\
\text { American Educational } \\
\text { Research Association }\end{array}$ & $\begin{array}{l}\text { The Relationship between } \\
\text { High School Mathematics } \\
\text { Classroom Environment and } \\
\text { Student Self-Handicapping }\end{array}$ \\
\hline 2002 & $P$ & Suíça & $\begin{array}{l}\text { Sex Roles: A Journal of } \\
\text { Research }\end{array}$ & $\begin{array}{l}\text { Blatant Stereotype Threat and } \\
\text { Women's Math Performance: } \\
\text { Self-Handicapping as a } \\
\text { Strategic Means to Cope with } \\
\text { Obtrusive Negative } \\
\text { Performance Expectations }\end{array}$ \\
\hline 2002 & $P$ & $\begin{array}{l}\text { Estados } \\
\text { Unidos }\end{array}$ & $\begin{array}{c}\text { Journal of Educational } \\
\text { Psychology }\end{array}$ & $\begin{array}{l}\text { The Classroom Environment } \\
\text { and Students' Reports of } \\
\text { Avoidance Strategies in } \\
\text { Mathematics: A Multimethod } \\
\text { Study }\end{array}$ \\
\hline 2003 & $P$ & $\begin{array}{l}\text { Estados } \\
\text { Unidos }\end{array}$ & $\begin{array}{c}\text { Elementary School } \\
\text { Journal }\end{array}$ & $\begin{array}{c}\text { Teacher Discourse and Sixth } \\
\text { Graders' Reported Affect and } \\
\text { Achievement Behaviors in Two } \\
\text { High-Mastery/High- } \\
\text { Performance Mathematics } \\
\text { Classrooms }\end{array}$ \\
\hline 2004 & $P$ & Canadá & $\begin{array}{l}\text { McGill Journal of } \\
\text { Education }\end{array}$ & $\begin{array}{l}\text { Associations between } \\
\text { Students' Perceptions of } \\
\text { Mathematics Classroom } \\
\text { Environment and Self- } \\
\text { Handicapping in Australian } \\
\text { and Canadian High Schools }\end{array}$ \\
\hline 2007 & $P$ & Inglaterra & $\begin{array}{c}\text { British Journal of } \\
\text { Educational Psychology }\end{array}$ & $\begin{array}{c}\text { Predicting Academic Self- } \\
\text { Handicapping in Different Age } \\
\text { Groups: The Role of Personal } \\
\text { Achievement Goals and Social } \\
\text { Goals }\end{array}$ \\
\hline 2012 & $P$ & $\begin{array}{l}\text { Estados } \\
\text { Unidos }\end{array}$ & $\begin{array}{c}\text { Community College } \\
\text { Review }\end{array}$ & $\begin{array}{l}\text { Achievement Goal Orientations } \\
\text { of Community College } \\
\text { Mathematics Students and the } \\
\text { Misalignment of Instructor's } \\
\text { Perceptions }\end{array}$ \\
\hline 2012 & $P$ & $\begin{array}{l}\text { Estados } \\
\text { Unidos }\end{array}$ & $\begin{array}{l}\text { European Journal of } \\
\text { Psychological } \\
\text { Assessment }\end{array}$ & $\begin{array}{l}\text { Measurement of metacognitive } \\
\text { knowledge of self, task, and } \\
\text { strategies in mathematics }\end{array}$ \\
\hline
\end{tabular}

Fonte: autores.

Das dez pesquisas selecionadas, entre artigos em periódicos ou apresentados em eventos, a mais antiga é de 1993 e a mais recente de 2012. Os anos com mais 
publicações foram 2002 e 2012 ( $n=2$ para ambos). Nenhuma publicação foi encontrada de 1994 a 1999, 2005 a 2006 e nem de 2008 a 2010. A única publicação de 2011 foi descartada pois corresponde à mesma publicação dos autores no ano de 2012, a qual se optou por utilizar. A maior parte foi publicada em periódicos dos Estados Unidos ou em eventos na área de Psicologia que ocorreram nesse país. Do total, 3 trabalhos foram publicados em anais de eventos e 7 foram publicados em periódicos. A seguir, os trabalhos encontrados serão sucintamente descritos, em ordem cronológica, do mais antigo ao mais recente.

A pesquisa de Steinhauer, Hymel e Woody (1993) teve como objetivo examinar se os indivíduos utilizam estratégias autoprejudiciais para proteger uma percepção geral ou global de si mesmos ou para proteger as percepções dos outros sobre suas competências em Matemática. Os participantes foram 495 estudantes do ensino médio. As percepções dos alunos sobre sua competência matemática foram avaliadas por meio de quatro escalas de competência autorrelatadas e a competência real foi medida por meio de dois testes aritméticos padronizados. Ao contrário do que se tinha como hipótese, os estudantes que superestimavam suas capacidades em Matemática não utilizaram estratégias autoprejudiciais antes dos testes, se esforçando da melhor forma possível para realizar o que havia sido proposto. Contudo, escolheram questões mais fáceis, o que parece evidenciar que eles tinham consciência de que suas capacidades estavam aquém daquelas declaradas. Por outro lado, aqueles que subestimavam suas competências utilizaram estratégias autoprejudiciais para evitar que suas baixas capacidades pudessem ser reveladas. Foi interessante observar que os dados deste estudo contrariaram a hipótese de que o senso de autoproteção estimula o uso de estratégias autoprejudiciais, o que pode ser constatado no caso daqueles que superestimavam suas competências.

A pesquisa de Brownlow, Rogers e Jacobi (2000) examinou a influência do gênero e de vários fatores históricos e de personalidade na ansiedade em relação à ciência, compreendida como o medo que muitos alunos brilhantes e capazes têm dos estudos científicos, nos quais a Matemática está incluída. Esse medo traz consigo o sentimento de incapacidade diante das demandas rumo ao aprendizado dessas ciências. Os participantes foram 87 estudantes matriculados em uma pequena faculdade do sudeste dos Estados Unidos, sendo 50 mulheres e 37 homens. Todos 
eram de origem caucasiana e de classe média. Para a coleta de dados, utilizou-se a Escala de Ansiedade Científica (MALLOW, 1994) aplicada aos participantes no início do semestre, antes de quaisquer exames. Esta escala inclui 44 afirmações sobre tarefas acadêmicas avaliadas em uma escala de 5 pontos ( 1 - de jeito nenhum a 5 - muito), de acordo com o nível de ansiedade que cada uma provoca. A soma dos itens produziu uma medida de ansiedade em relação à ciência de cada participante. Os resultados mostraram que alunos com alta ansiedade relativa à ciência fizeram menos cursos de ciências na faculdade, tiveram notas mais baixas em testes e relataram que seus professores de ciências do ensino médio não foram úteis. Além disso, eram mais perfeccionistas, sugerindo que a ansiedade pela ciência pode ser resultado do desejo de evitar tarefas que nem sempre podem ser concluídas com sucesso. Especificamente em relação à Matemática, as mulheres obtiveram melhores notas, embora não houvesse diferença entre os gêneros em termos de preparação. Os homens se mostraram mais propensos à adoção de estratégias autoprejudiciais com a tendência de culpar agentes externos pelos seus maus desempenhos.

O objetivo da pesquisa de Dorman, Adams e Ferguson (2001) foi investigar a a relação entre o ambiente da sala de aula de Matemática e o uso de estratégias autoprejudiciais. A amostra, composta por 3.602 estudantes de 29 escolas do ensino médio australianas, canadenses e britânicas, respondeu a um questionário que avaliou as percepções dos alunos sobre o ambiente da sala de aula de Matemática, as estratégias autoprejudiciais e a eficácia acadêmica. Os resultados mostraram que o ambiente da sala de aula foi responsável por proporções apreciáveis de variância na ocorrência das estratégias autoprejudiciais e na percepção de eficácia acadêmica. Dimensões afetivas do ambiente de sala de aula foram associadas a níveis reduzidos de uso de estratégias autoprejudiciais.

Keller (2002) realizou uma pesquisa cujo objetivo foi identificar o impacto de pertencer a um grupo de estereótipo negativo na adoção de estratégias autoprejudiciais e no desempenho posterior em Matemática. Os participantes foram 75 estudantes do ensino médio, sendo 37 mulheres e 38 homens, aos quais foi aplicado um teste de Matemática com 20 questões. O estudo se baseou na Teoria de Ameaça dos Estereótipos. Essa teoria parte do pressuposto que, ao acreditar que pertence a um grupo alvo de um estereótipo negativo, o indivíduo, quando 
confrontado com uma tarefa relacionada a este estereótipo, terá seu desempenho reduzido. Os resultados mostraram que as mulheres, na condição de grupo de esteréotipo negativo, tiveram um desempenho inferior em comparação com os homens. Os dados apontaram ainda para o fato de que a ameaça de estereótipo pode induzir a necessidade de se proteger de atribuições negativas, estando em consonância com outros estudos que documentaram o uso elevado de estratégias autoprejudiciais em situações de expectativas de desempenho negativas.

Analisar a relação entre o ambiente de aprendizagem (percepções sobre a coerência entre objetivo da aula e o discurso instrucional dos professores) e o uso relatado de estratégias autoprejudiciais pelos alunos, em salas de Matemática, foi o objetivo da pesquisa de Turner et al. (2002). Os participantes foram 1.092 alunos (52\% mulheres, 70\% euro-americanos e 30\% afro-americanos). De acordo com os autores, a principal contribuição do estudo foi fornecer um exame qualitativo das práticas de discurso dos professores para ajudar a explicar por que os alunos usam estratégias autoprejudiciais. Nesse sentido, o estudo mostrou que o uso de estratégias autoprejudiciais foi significativamente menor em salas de aula nas quais o discurso do professor vai na direção de apoiar instrucional e motivacionalmente os estudantes, em um ambiente em que são valorizados o aprendizado e a compreensão, mas também o esforço e o prazer. Além disso, professores com este perfil mostram aos estudantes que também podem aprender com os erros e que ter dúvidas, fazer perguntas e procurar ajuda fazem parte do processo de aprendizagem.

No artigo intitulado “Teacher Discourse and Sixth Graders' Reported Affect and Achievement Behaviors in Two High-Mastery/High-Performance Mathematics Classrooms" (TURNER et al., 2003), três dos autores são os mesmos do anteriormente apresentado. Este artigo retrata uma pesquisa cujo objetivo foi examinar a relação entre a natureza do discurso do professor e os relatos de um grupo de 34 estudantes de duas turmas da sexta série sobre comportamentos dos professores que contribuem, ou não, para a adoção de estratégias autoprejudiciais. De modo semelhante ao estudo anterior realizado em amostra maior e mais representativa, constatou-se que os estudantes da sala de aula em que havia apoio constante de incentivo à autonomia e estratégias de motivação intrínseca, afeto positivo e 
colaboração relataram menor adoção de estratégias autoprejudiciais quando comparados àqueles da turma sem este tipo de apoio.

Dorman e Ferguson (2004) realizaram um estudo orientado a investigar a relação entre o ambiente da sala de aula de Matemática e o uso de estratégias autoprejudiciais. Os participantes foram 2.006 estudantes, australianos e canadenses. Para a coleta de dados foram aplicados questionários aos estudantes para acessar suas percepções sobre o ambiente de sala de aula e sua relação com o uso de estratégias autoprejudiciais. De acordo com os autores, aspectos afetivos da relação professoraluno foram associados a níveis reduzidos no uso de estratégias autoprejudiciais. Nas salas de aula em que o professor prestava mais assistência aos estudantes, apoiandoos instrucional e emocionalmente, havia menor predisposição ao uso de estratégias autoprejudiciais. O estudo também mostrou que a relação entre ambiente de sala de aula e uso de estratégias autoprejudiciais nas salas de aula canadenses e australianas era muito semelhante. Não houve diferenças significativas de gênero na relação entre ambiente de sala de aula e uso de estratégias autoprejudiciais.

No estudo de Leondari e Gonida (2007), o objetivo foi investigar a associação entre objetivos pessoais, objetivos sociais, consequências futuras e o uso relatado de estratégias de autoprejudiciais, além de diferenças entre níveis escolares e de gênero em relação ao uso de estratégias autoprejudiciais no domínio da Matemática, entre estudantes do Ensino fundamental e médio. Os participantes foram 702 estudantes dos ensinos fundamental e médio de cinco escolas públicas de áreas urbanas da Grécia. Desse total, 368 eram meninos e 334 eram meninas, 504 do ensino fundamental e 198 do ensino médio. Todos os participantes foram convidados a responder um questionário em relação ao domínio da matemática. O fato de a pesquisa ter se limitado ao domínio da Matemática se justifica, pois há evidências de que as crenças e percepções relacionadas à motivação tendem a ser diferenciadas de acordo com os domínios de realização e áreas do conhecimento. Em outras palavras, o uso de estratégias autoprejudiciais pode variar de acordo com a disciplina. O estudo concluiu que estudantes com notas mais baixas em matemática tendiam a fazer mais uso de estratégias autoprejudiciais do que estudantes com notas mais altas. O uso de estratégias autoprejudiciais também se relacionou com a meta de agradar a outras pessoas significativas, particularmente entre estudantes que tinham assumido como 
meta alcançar bom desempenho para agradar os pais e professores. De acordo com os autores, este achado está em consonância com dados de outras pesquisas que relacionam a procrastinação às expectativas irreais dos pais. Além disso, os resultados mostraram que não houve diferença significativa entre os níveis de ensino (fundamental, médio) e nem de gênero, no que se refere ao uso de estratégias autoprejudiciais.

Investigar as orientações às metas de realização de alunos de matemática e as percepções de seus professores sobre essas metas foi o objetivo do estudo de Mesa (2012). Os participantes foram 777 estudantes matriculados em cursos de reforço e universitários que cursavam matemática em uma grande faculdade comunitária de um subúrbio no Meio-Oeste americano, além de 25 professores. Os resultados indicaram que os estudantes estão interessados em desenvolver competências, esperam e acreditam que podem lidar com trabalhos desafiadores, evitam usar estratégias autoprejudiciais e exibem um autoconceito matemático positivo. No entanto, as entrevistas com docentes das disciplinas em que os alunos estavam matriculados revelaram que estes professores tinham uma perspectiva mais negativa em relação aos seus estudantes. Esta discrepância sugere que os instrutores podem não estar aproveitando a alta confiança e motivação para aprender que seus alunos trazem para a sala de aula de matemática.

Efklides e Vlachopoulos (2012) investigaram a validade do Questionário de Conhecimento Metacognitivo em Matemática, que explora: (1) o conhecimento metacognitivo de si mesmo (facilidade / fluência versus dificuldade / falta de fluência que a pessoa teve no passado em noções matemáticas básicas); (2) o conhecimento metacognitivo de tarefas (tarefas matemáticas fáceis / baixas versus tarefas difíceis), e (3) o conhecimento metacognitivo de estratégias (estratégias cognitivas / metacognitivas, estratégias de aumento de competência e estratégias de evitação, que servem para lidar com falta de fluência no processamento de tarefas matemáticas). $\mathrm{O}$ questionário foi administrado primeiro a 311 alunos do ensino médio ( $7^{\mathrm{a}}$ e $9^{\mathrm{a}}$ séries) e depois a 214 estudantes universitários da Grécia para validação cruzada. Em ambas as amostras, houve correlação entre os fatores e as medidas de autoconceito em matemática e habilidade matemática. Especificamente em relação às estratégias de evitamento (avoidance strategies), os pesquisadores concluíram que o reconhecimento 
das dificuldades em Matemática levou à adoção desse tipo de estratégia por parte dos estudantes.

Considerando as dez pesquisas apresentadas, em sete delas os participantes foram estudantes de turmas de anos escolares que correspodem ao ensino básico no Brasil (Ensinos Fundamental e Médio). Em um estudo, os participantes foram do curso superior. Em outro, o público era misto, incluindo estudantes do ensino básico e do ensino superior. Um dos estudos não informava o nível de escolaridade no qual se encontravam os participantes. De todo modo, os estudos revistos evidenciaram que os participantes foram estudantes que já atingiram maturidade cognitiva para fazer uso de estratégias autoprejudiciais, sendo capazes de compreender a diferença entre inteligência e esforço, tal como mencionado por Garcia et al. (1995 apud MIDGLEY; ARUNKUMAR; URDAN, 1996).

Percebeu-se, no exame dos trabalhos, alguns aspectos que os aproximam, particularmente em relação aos objetivos e aos resultados encontrados. A partir disso, foram elaboradas categorias de análise para possibilitar um diálogo entre pesquisas selecionadas e outras pesquisas que abordam as estratégias autoprejudiciais. Os artigos revisitados são analisados à luz destas categorias, a seguir.

Afetividade e ambiente escolar - Alguns estudos concluíram que aspectos afetivos concorrem para maior utilização, ou não, de estratégias autoprejudiciais em sala de aula (DORMAN; ADAMS; FERGUSON, 2001; TURNER et al., 2002; TURNER et al., 2003; DORMAN; FERGUSON, 2004). Considera-se importante ressaltar que, em todos esses estudos, o ambiente da sala de sala teve destaque como elemento que contribuiu para maior ou menor utilização de estratégias autoprejudiciais. Neste ambiente, a figura do professor foi colocada em relevo, como aquele cujos discursos e práticas se mostraram eficazes para evitar o uso de estratégias autoprejudiciais. $\mathrm{O}$ apoio motivacional aos estudantes parece ter reduzido a ocorrência dessas estratégias. Nesse sentido, o estudo de Şahin e Çoban (2020) concluiu que em um clima escolar positivo, no qual os comportamentos de professores são orientados pelo desejo de criar um ambiente seguro e no qual os estudantes tenham possibilidade de alcançar sucesso, diminui as chances de ocorrência de usos de estratégias autoprejudiciais. Além disso, os estudantes que não 
adotam essas estratégias, ou as adotam com pouca frequência, tendem a obter melhores desempenhos.

Embora o ambiente escolhido para as pesquisas tenha sido a sala de aula de Matemática, a afetividade tem sido considerada por vários pesquisadores como fundamental na relação para criar um clima propicio à aprendizagem (RIBEIRO, 2010; ECCIUS-WELLMANN; IBARRA-GONZALEZ, 2020). A afetividade é importante, inclusive, para a aprendizagem cognitiva (CODO; GAZZOTI, 1999). Contudo, os problemas enfrentados pelos estudantes em Matemática, considerada como muito difícil, destinada apenas aos mais capacitados, talvez contribuam para que o papel da afetividade na relação professor-aluno seja ainda mais relevante no caso dessa disciplina, de modo a amenizar o uso de estratégias autoprejudiciais, como concluíram os estudos apresentados.

A afetividade pode ser comprrendida sob várias perspectivas: atitudes e valores, comportamento moral e ético, desenvolvimento pessoal e social, motivação, interesse e atribuição, ternura, interrelação, empatia, constituição da subjetividade, sentimentos e emoções (RIBEIRO, 2010). Dessa forma, a autoestima, considerada como um sentimento que figura nas transações afetivas que ocorrem em sala de aula, tem impacto na adoção de estratégias autoprejudiciais pelos estudantes. Estudantes com baixa autoestima tendem a utilizar com maior frequência as estratégias autoprejudiciais como instrumento de autoproteção do seu valor enquanto pessoa (LIMA, 2016; BORUCHOVITCH, 2014; YAVUZER, 2015). Aqui, mais uma vez, as práticas do professor podem contribuir para melhorar a autoestima dos estudantes como alternativa possível para evitar o uso destas estratégias nocivas à aprendizagem.

Estes estudos evidenciam, para além dos aspectos afetivos, que a sala de aula é um espaço efetivo para se investigar a ocorrência de estratégias autoprejudiciais, corroborando as ideias de Sameer Babu e Selvamari (2018), que consideram que o ambiente escolar, em particular a sala de aula, é visto como bastante adequado para examinar essas estratégias, uma vez que os tipos de atividades nele desenvolvidos suscitam nos alunos questionamentos constantes acerca de suas capacidades e condições de desempenhar as tarefas propostas com sucesso.

Gênero - Em três dos estudos revistos, os resultados encontrados trazem à tona a discussão sobre gênero, no que se refere ao uso de estratégias autoprejudiciais 
em Matemática. Em um deles (BROWNLOW; ROGERS; JACOBI, 2000), os dados evidenciaram que os homens se mostraram mais propensos à adoção de estratégias autoprejudiciais com a tendência de culpar agentes externos pelos maus desempenhos. Nos outros dois estudos selecionados (DORMAN; FERGUSON, 2004; LEONDARI; GONIDA, 2007), o mesmo não ocorreu. De acordo com os autores, os resultados mostraram que não houve diferença significativa de gênero, no que se refere ao uso dessas estratégias.

O resultado dos dois primeiros estudos está de acordo com aquele encontrado por Yu e McLellan (2019), no qual constatou-se que os meninos adotam com maior frequência estratégias autoprejudiciais porque têm o desejo mais forte de preservar sua imagem nos domínios acadêmico e social. Entretanto, difere do resultado da pesquisa de Yavuzer (2015), no qual a autora encontrou, como parte dos resultados, maior propensão das mulheres ao uso de estratégias autoprejudiciais. Embora os estudos de Yu e McLellan (2019) e Yavuzer (2015) não tenham tido foco na Matemática, seus achados são importantes para comparação.

Resultados diferentes quanto ao uso de estratégias autoprejudiciais por homens e mulheres certamente são importantes para corroborar os apontamentos feitos por Midgley, Arunkumar e Urdan (1996) sobre o fato de que o uso de estratégias autoprejudiciais se relaciona às características particulares dos estudantes, como suas motivações gerais, atitudes em relação à aprendizagem, ao modo como se veem e se descrevem como alunos e, podemos acrescentar, ao ambiente em que estão inseridos. Em outras palavras, pode ser que o uso dessas estratégias esteja mais relacionado às idiossincrasias de cada pessoa, incluindo o meio em que vive, do que ao seu gênero.

O estudo de Dorman e Ferguson (2004) foi realizado com estudantes canadenses e australianos. O de Leondari e Gonida (2007) com estudantes gregos. Yu e McLellan (2019) tiveram em sua pesquisa participantes ingleses e Yavuzer (2015) estudantes turcos. Esta variedade de nacionalidades pressupõe, também, uma variedade de culturas, valores, crenças, modos de ser e fazer que, muito provavelmente, afetam o comportamento das pessoas. Cabe destacar que, além das particularidades de cada pessoa, o ambiente em que ela se insere tem forte influência na sua decisão pelo uso ou não de estratégias autoprejudiciais. 
Embora os resultados de pesquisas que relacionam gênero e uso de estratégias autoprejudiciais não sejam unânimes em relação a um uso mais intenso dessas estratégias pelos meninos, isso pode ser uma tendência muito comum. A justificativa para esta afirmação está no fato de que os meninos parecem possuir um desejo mais forte de preservar sua imagem nos domínios acadêmico e social do que as meninas. No domínio acadêmico, o uso de estratégias autoprejudiciais serve para evitar que venham à tona a falta de competência nos conteúdos (de disciplinas específicas ou não). No âmbito social, o uso mais frequente dessas estratégias é resultado de suas maiores preocupações com o status social, ou seja, a imagem que os outros têm dele (YU; McLELLAN, 2019). Considerando as inconsistências existentes quanto ao impacto do gênero no uso das estratégias autoprejudiciais e a importância desta variável, recomenda-se que a pesquisa futura se volte a desvendâ-las com mais precisão, já que os dados a serem obtidos têm importantes implicações psicoeducacionais e podem ser mais bem instrumentalizados a favor do gênero que mais prejudica a sua aprendizagem, se assim ficar conhecido.

Construtos ligados ao self - Em dois estudos, os autores apresentam o autoconceito como um construto avaliado nos estudantes. Mesa (2012) concluiu que os participantes da pesquisa estavam interessados em desenvolver competências, acreditavam em sua capacidade para lidar com tarefas desafiadoras no âmbito da Matemática, evitavam o uso de estratégias autoprejudiciais e exibiam um autoconceito matemático positivo. Efklides e Vlachopoulos (2012) concluíram que houve correlação entre as medidas de autoconceito em matemática e habilidade matemática e que em relação às estratégias de evitamento (avoidance strategies), o reconhecimento das dificuldades em Matemática levou à sua adoção. Como o autoconceito é um conjunto de crenças e representações a respeito de si, que norteiam o comportamento do indivíduo e permitem que esse desempenhe diversos papéis em sua vida (OLIVEIRA, 2000), e considerando que o uso de estratégias autoprejudiciais pode ser tido como um comportamento do indivíduo antes de tarefas que ele teme, parece coerente a relação encontrada entre esses construtos.

As crenças de autoeficácia, embora não tenham sido discutidas diretamente como um fator que pode contribuir para o uso de estratégias autoprejudiciais, parecem permear as decisões de alguns estudantes quanto ao seu uso. As crenças de 
autoeficácia dizem respeito aos julgamentos da pessoa sobre suas capacidades para realizar tarefas em um domínio específico (BANDURA, 1992; BZUNECK, 2001; PAJARES, 1996). Elas são menos gerais que o autoconceito. O estudo de Steinhauer, Hymel e Woody (1993), por exemplo, utilizou, dentre os instrumentos de coleta de dados, dois testes matemáticos. Aqueles que superestimavam suas capacidades realizaram os testes e não lançaram mão de estratégias autoprejudiciais, mas escolheram as questões mais fáceis. Por outro lado, aqueles que subestimavam suas competências utilizaram estratégias autoprejudiciais para evitar que suas baixas capacidades pudessem vir à tona. A escolha por tarefas mais fáceis por parte daqueles que superestimavam suas capacidades pode ser um indício de que possuíam crenças de autoeficácia pouco robustas quanto à sua capacidade de realizar as questões mais difíceis. Isso parece ainda mais coerente quando se leva em conta que as crenças de autoeficácia "se referem de modo específico a peculiaridades da situação, [...] e ações a serem implementadas numa tarefa analisada em detalhe" (BZUNECK, 2001, p. 117). Sendo assim, embora essa conclusão não tenha sido do estudo de Steinhauer, Hymel e Woody (1993), parece relevante aventar que o comportamento dos estudantes diante dos testes possa ter sido afetado também por suas crenças de autoeficácia. Tal como mostra a literatura, o desempenho escolar e acadêmico é resultante de vários fatores cognitivos, metacognitivos, motivacionais (metas de realização, atribuições de causalidade, autoeficácia), afetivos e sociais (BORUCHOVITCH， 2014; ZIMMERMAN; SCHUNK， 2011; SCHUNK; GREENE, 2018).

\section{Considerações finais}

A adoção de estratégias autoprejudiciais tem sérias implicações para o rendimento dos estudantes em todas as disciplinas. Particularmente em relação à Matemática, considerada como difícil e destinada a poucos, o uso de estratégias desse tipo pode ser uma saída para muitos estudantes se autoprotegerem dos julgamentos dos outros sobre suas capacidades falhas. O presente artigo nos leva à reflexão sobre vários pontos. Um deles é o reduzido número de pesquisas com foco no uso de estratégias autoprejudiciais em Matemática, mesmo em âmbito internacional. No que 
concerne às pesquisas brasileiras sobre a temática, essa situação é ainda mais preocupante, pois embora a Matemática seja uma disciplina temida por muitos, as pesquisas que investigam estratégias autoprejudiciais em Matemática praticamente inexistem no cenário acadêmico brasileiro.

Outra reflexão necessária diz respeito ao importante papel que devemos atribuir ao ambiente social, como um elemento que influencia de modo decisivo o uso, ou não, das estratégias autoprejudiciais. Não nos referimos somente ao ambiente de sala de aula. A escola e a universidade estão inseridas em um contexto social maior, cujos acontecimentos afetam de forma direta o comportamento dos estudantes. Alguns dos estudos ora revisitados colocaram em evidência essa importância. $\mathrm{O}$ fato, por exemplo, de meninos apresentarem tendência maior ao uso de estratégias autoprejudiciais comparados às meninas tem relação com ambientes competitivos, de disputa de poder, de luta por pertencimento muito comum em quase todas as culturas. Os meninos parecem se preocupar mais em mostrar suas capacidades escolares e acadêmicas, bem como com a preservação de seu status social (YU; McLELLAN, 2019). Isso não é, naturalmente, uma generalização, mas ilustra a importância do ambiente na adoção e na prevenção do uso de comportamentos autoprejudiciais.

Esta discussão tem implicações educacionais importantes, sobretudo em relação à atuação dos professores em sala de aula. Em contextos nos quais os professores fazem maior uso de práticas instrucionais orientadas para o desempenho (por exemplo, ênfase nas notas, capacidade e comparação social), os estudantes têm maior tendência ao uso de estratégias autoprejudiciais para se proteger de um mau resultado, que pode não ser bem-visto pelos professores e colegas. Professores com práticas que consideram aspectos afetivos, como valorização dos esforços individuais, independentemente do gênero, motivação extrínseca, ações para melhorar a autoestima e incrementar crenças de autoeficácia, dentre outros, podem contribuir para que os estudantes adotem menos estratégias autoprejudiciais, como mostraram alguns estudos.

\section{Referências}

BANDURA, A. Social Foundantions of Thought \& Action - A Social Cognitive Theory. Englewood Cliffs: Prentice Hall, 1986. 
BANDURA, A. The self system in reciprocal determinism. American Psychologist, v. 33, n. 4, p. 344-358, 1978.

BANDURA, A. Exercise of personal agency through the self-efficacy mechanism. In: SCHWARZER, R. (Ed.). Self-efficacy: Thought control of action. [S.l.]: Hemisphere Publishing Corp, 1992. p. 3-38.

BERGLAS, S.; JONES, E. E. Drug choice as a self-handicapping strategy in response to noncontingent success. Journal of Personality and Social Psychology, v. 36, n. 4, p. 405-417, 1978.

BOEKAERTS, M. Subjective competence, appraisals and self-assessment. Learning and Instruction, v. 1, n. 1, p. 1-17, 1991.

BOEKAERTS, M. The adaptable learning process: initiating and maintaining behavioural change. Applied Psychology, v. 41, n. 4, p. 377-397, 1992.

BORUCHOVITCH, E. Self-regulated learning: contributions of the educational psychology to teacher education. Psicol. Esc. Educ., Maringá, v. 18, n. 3, p. 401-409, dez. 2014.

BROWNLOW, S.; ROGERS, M. I.; JACOBI, T. Science Anxiety as a Function of Personality, Gender Roles, Experience with Science. Sex Roles, v. 42, p. 139-141, 1997.

BZUNECK, J. A. As crenças de auto-eficácia e o seu papel na motivação do aluno. In: BORUCHOVITCH, E.; BZUNECK, J. A. (Eds.). A motivação do aluno: Contribuições da psicologia contemporânea. Petrópolis: Vozes, 2001. p. 116-133.

CHEN, Z.; SUNG, K.; WANG, K. Self-esteem, achievement goals, and self-handicapping in college physical education. Psychological Reports, v. 121, n. 4, p. 690-704, 2017.

CODO, W.; GAZZOTTI, A. A. Trabalho e afetividade. In: CODO, W. (Dir.). Educação, carinho e trabalho. 3. ed. Petrópolis: Vozes, 1999. p. 48-59.

COUDEVYLLE, G. R.; BOULEY-ESCRIVA, G.; FINEZ, L.; EUGÈNE, K.; ROBIN, N. An experimental investigation of claimed self-handicapping strategies across motivational climates based on achievement goal and self-determination theories. Educational Psychology: an international journal of experimental educational psychology, v. 40, n. 8, p. 1002-2021, 2020.

COVINGTON, M. V. Goal Theory, Motivation, and School Achievement: An Integrative Review. Annual Review of Psychology, v. 51, p. 171-200, 2000.

DORMAN, J. P.; ADAMS, J. E.; FERGUSON, J. M. The Relationship between High School Mathematics Classroom Environment and Student Self-Handicapping. In: ANNUAL MEETING OF THE AMERICAN EDUCATIONAL RESEARCH ASSOCIATION, 2001, Seattle. Proceedings... Seattle: [s.n.], 2001. p. 1-22.

DORMAN, J. P.; FERGUSON, J. M. Associations between Students' Perceptions of Mathematics Classroom Environment and Self-Handicapping in Australian and Canadian High Schools. McGill Journal of Education, v. 39, n. 1, p. 69-87, 2004.

EFKLIDES, A. Interactions of metacognition with motivation and affect in self-regulated learning: the MASRL model. Educational Psychology, v. 46, n. 1, p. 6-25, 2011. 
EFKLIDES, A.; VLACHOPOULOS, S. P. Measurement of metacognitive knowledge of self, task, and strategies in mathematics. European Journal of Psychological Assessment, v. 28, n. 3, p. 227-239, 2012.

ECCIUS-WELLMANN, C.; IBARRA-GONZALEZ, K. P. Dependencia de la calificación de una evaluación diagnóstica en matemáticas con aspectos afectivos por la comisión de errores. Bolema, Rio Claro, v. 34, n. 67, p. 544-563, 2020.

GANDA, D. R.; BORUCHOVITCH, E. Intervenção em autorregulação da aprednizagem com alunos do ensino superior: análise da produção científica. Estudos interdisciplinares em Psicologia, v. 10, n. 3, p. 3-25, 2019.

GANDA, D. R.; BORUCHOVITCH, E. As atribuições de causalidade e as estratégias autoprejudiciais de alunos do curso de Pedagogia. Psico-USF, v. 21, n. 2, p. 331-340, 2016.

GOMES, M.; BORUCHOVITCH, E. A promoção da aprendizagem autorregulada-O protagonismo de professores e alunos em perspectiva. In: FRISON, L. M. B.; BORUCHOVITCH, E. (Org.). Autorregulação da aprendizagem: cenários, desafios e perspectivas para o contexto educativo. Petropólis: Editora Vozes, 2020. v. 1, p. 87-113.

HADWIN, A.; JÄRVELÄ, S.; MILLER, M. Self-regulation, co-regulation, and shared regulation in collaborative learning environments. In: SCHUNK, D. H.; GREENE, J. A. (Eds.). Educational psychology handbook series: Handbook of self-regulation of learning and performance. New York: Routledge, 2018. p. 83-106.

HIRT, E. R.; DEPPE, R. K.; GORDON, L. J. Self-reported versus behavioral selfhandicapping: empirical evidence for a theoretical distinction. Journal of personality and social psychology, v. 61, n. 6, p. 981-991, 1991.

KELLER, J. Blatant stereotype threat and women's math performance: Self-handicapping as a strategic means to cope with obtrusive negative performance expectations. Sex Roles: $A$ Journal of Research, v. 47, n. 3/4, p. 193-198, 2002.

LEONDARI, A.; GONIDA, E. Predicting academic self-handicapping in different age groups: the role of personal achievement goalsand social goals. British Journal of Educational Psychology, v. 77, p. 595-611, 2007.

LIMA, C. M. O uso de estratégias autoprejudiciais nos cursos de licenciatura. Orientadora: Evely Boruchovitch. Trabalho de Conclusão de Curso (Graduação em Pedagogia) — Faculdade de Educação, Universidade Estadual de Campinas, Campinas, 2016.

MALLOW, J. V. Gender-related science anxiety: a first binational study. Journal of Science Education and Technology, v. 3, p. 227-238, 1994.

MCCASLIN, M.; GOOD. T. L. The informal curriculum. In: BERLIN, D. C.; CALFEE, R. C. (Orgs.). Handbook of educational psychology. New York: Simon \& Schuster Macmillan, 1996. p. $622-670$.

MESA, V. Achievement Goal Orientations of Community College Mathematics Students and the Misalignment of Instructor Perceptions. Community College Review, v. 40, n. 1, p. 4674, 2012. 
MIDGLEY, C.; ARUNKUMAR, R.; URDAN, T. C. If I don't well tomorrow, there's a reason: predictors of adolescentes. Use of academic self-handcapping srategies. Journal of Educational Psychology, v. 88, n. 3, p. 423-434, 1996.

MIRANDA, L. C.; BORUCHOVITCH, E.; GANDA, D. R. Contributos para a validação da escala de estratégias autoprejudiciais em alunos do ensino secundário português. Revista Amazônica, v. XIX, n. 2, p. 8-22, 2017.

OLIVEIRA, G. C. Autoconceito do adolescente. In: SISTO, F. F.; OLIVEIRA, G. C.; Oliveira.; FINI, L. D. T. Leituras de psicologia para formação de professores. Petrópolis: Vozes, 2000. p. $58-69$.

PAJARES, F. Self-efficacy beliefs in academic settings. Review of Educational Research, v. 66, p. 543-578, 1996.

PEKRUN, R. Control-value theory: a social cognitive approach. In: LIEM, G. A. D.; McINERNEY, D. M. (Orgs.). Big theories revisited 2. Charlotte: Information Age, 2018. p. 165192.

PEKRUN, R. The control-value theory of achievement emotions: assumptions, corolaries, and implications for educational research and practice. Educational Psychology Review, v. 18, n. 4, p. 315-341, 2006.

PINTRICH, P. R. The role of goal orientation in self-regulated learning. In: BOEKAERTS, M.; PINTRICH, P.; ZEIDNER, M. (Orgs.). Handbook of self-regulation. San Diego, CA: Academic Press, 2000. p. 13-39.

PINTRICH, P. A conceptual framework for assessing motivation and self-regulated learning in college students. Educational Psychology Review, v. 16, n. 4, p. 385-407, 2004.

PRANKE, A.; FRISON, L. M. B. Potencialização da Aprendizagem Autorregulada de Bolsistas do PIBID/UFPel do curso de Licenciatura em Matemática através de Oficinas Pedagógicas. Bolema, Rio Claro, v. 29, n. 51, p. 223-240, 2015.

PRAPAVESSIS, H.; GROVE, J. R. Self-Handicapping and Self-Esteem. Journal of applied sport Psychology, v. 10, p. 175-184, 1998.

RIBEIRO, M. L. A afetividade na relação educativa. Estud. Psico, Campinas, v. 27, n. 3, p. 403-412, 2010.

ŞAHIN, F.; ÇOBAN, Ö. Effect of School Climate, Students 'Self-Handicapping Behaviors Effect of School Climate, Students 'Self-Handicapping Behaviors and Demographic Characteristics on Students' Achievement and Demographic Characteristics on Students' Achievement. Inquiry in Education, v. 12, n. 2, p. 1-20, 2020.

SAMEER, B. M.; SELVAMARI, S. How does academic self-handicapping relate o achievement in mathematics?: a small scale study among indian school chult. Educational quest: an international journal of education and applied social Science, v. 9, n. 3, p. 233-238, 2018.

SCHUNK, D. H. Self-regulation through goal setting. Greensboro, NC: ERIC Clearinghouse on Counseling and Student Service, 2001. 
SCHUNK, D.; ZIMMERMAN, B. Motivation and self-regulated learning: Theory, research and applications. New York: Lawrence Erlbaum Associates, 2008.

SCHUNK, D.; GREENE, J. A. Handbook of self-regulation of learning and performance. 2. ed. New York: Routledge, 2018.

SHEPPARD, J. A.; ARKIN, R. M. Determinants of self-handi-capping: task importannce and effects of preexisting handicaps of self-generated handicaps. Personality and Social Psychology Bulletin, v. 15, n. 1, p. 101-112, 1989.

STEINHAUER, A.; HYMEL, S.; WOODY, E. Z. An Assessment of the Self-Protective Function of Self-Handicapping. In: BIENNIAL MEETING OF THE SOCIETY FOR RESEARCH IN CHILD DEVELOPMENT, 1993, Nova Orleans. Proceedings... [S.l.: s.n.], 1993. p. 1-18.

TORISU, E. M.; FERREIRA, A. C. A teoria social cognitiva e o ensino-aprendizagem da Matemática: considerações sobre as crenças de autoeficácia matemática. Ciências e Cognição, v. 14 , n. 3, p. 168-177, 2009.

TURNER, J. C. et al. Teacher Discourse and Sixth Graders' Reported Affect and Achievement Behaviors in Two High-Mastery/High-Performance Mathematics Classrooms. The Elementary School Journal, v. 103, n. 4, p. 357-382, 2003.

TURNER, J. C.; MIDGLEY, C.; MEYER, D. K.; GHEEN, M.; ANDERMAN, E. M.; KANG, Y.; PATRICK, H. The classroom environment and students' reports of avoidance strategies in mathematics: A multimethod study. Journal of Educational Psychology, v. 94, n. 1, p. 88-106, 2002.

URDAN, T.; MIDGLEY, C. Changes in the perceived classroom goal structure and pattern of adaptive learning during early adolescence. Contemporary Educational Psychology, v. 28, n. 4, p. 524-551, 2003.

URDAN, T.; MIDGLEY, C. Academic Self-Handicapping: What We Know, What more there is to learn. Educational Psychology Review, v. 13, n. 2, p. $114-138,2001$.

WINNE, P.H.; HADWIN, A. F. Studying as a self-regulated learning. In: HACKER, D. J.; DUNLOSKY, J.; GRAESSER, A. C. (Orgs.). Metacognition in educational theory and practice. Mahwah, NJ: Lawrence Erlbaum Associates, 1998. p. 277-304.

YAVUZER, Y. Investigating the Relationship between Self-Handicapping Tendencies, SelfEsteem and Cognitive Distortions. Educational Sciences: Theory and Practice, v. 15, n. 4, p. 879$890,2015$.

YU, J.; McLELLAN, R. Beyond academic achievement goals: the important of social achievement in explaining gender differences in self-handicapping. Learning and individual differences, v. 69, p. 33-44, 2019.

ZIMMERMAN, B. J. Attaining self-regulation: A social cognitive perspective. In: BOEKARTS, M.; PINTRICH, P. R.; ZEIDNER, M. (Orgs.). Handbook of self-regulation. San Diego, CA: Academic Press, 2000, p. 13-39.

ZIMMERMAN, B. J.; SCHUNK, D. H. Handbbok of self-regulation of learning and performance. Nova York: Routledge, 2011. 
ZUCKERMAN, M.; KIEFFER, S. C.; KNEE, C. R. Consequences of self-handicapping: Effects on coping, academic performance, and adjustment. Journal of Personality and Social Psychology, v. 74, n. 6, p. 1619-1628, 1998.

RECEBIDO: $18 / 05 / 2021$

APROVADO: $21 / 07 / 2021$
RECEIVED: 05/18/2021

APPROVED: 07/21/2021
RECIBIDO: $18 / 05 / 2021$

APROBADO: 21/072021 